\title{
Critical care nurses be aware: Lemierre's syndrome is on the rise
}

\author{
Roseanne Jane $\bullet$ BN MCCN \\ Clinical Educator ICU, Gold Coast Hospital, Qld \\ Patricia Johnson - RN Intensive Care Cert. BA MN (Hons) PhD \\ Lecturer and Convenor Critical Care Programs, Faculty of Nursing and Health \\ Griffith University, Gold Coast Campus, Bundall, Qld
}

Abstract: Lemierre's syndrome (LS) typically occurs in previously healthy young adolescents and young adults who become acutely ill following an attack of pharyngotonsillitis. Also known as post anginal sepsis, those afflicted develop pyrexia, rigours and multiple metastatic abscesses that lead to septic thrombophlebitis of the internal jugular vein. In the pre-antibiotic era this particularly virulent syndrome had a mortality rate in excess of $90 \%$, but since the introduction of antibiotics and the widespread treatment of throat infections, it has became almost unknown. However, due to a number of factors, including a reduction in the use of antibiotics for the treatment of sore throats, misdiagnosis and/or improvements in microbiology diagnostic techniques, several reports have indicated a resurgence of the condition. This has major ramifications for critical care nurses as $L S$ is still associated with significant morbidity and mortality.

This paper discusses the aetiology, pathophysiology, bacteriology, diagnosis and management aspects of this syndrome. A case study of a young woman is presented to illustrate the complexity of the condition, and highlight how early diagnosis and prompt initiation of appropriate intravenous antibiotic therapy ensured a favourable clinical outcome.

Jane R $\mathcal{E}$ Johnson P. Critical care nurses be aware: Lemierre's syndrome is on the rise. Australian Critical Care 2003; 16(4):126-132.

\section{INTRODUCTION}

In 1936, Lemierre, a French physician, described an acute syndrome characterised by oropharyngeal infection with septic thrombophlebitis of the internal jugular vein. He termed this condition post anginal septicaemia. Lemierre's syndrome (LS), also known as necrobaccillosis, is a systemic anaerobic bacterial infection usually associated with Fusobacterium necrophorum ${ }^{1 \cdot 3}$.

Prior to the usage of antibiotics to treat LS, this condition was associated with a mortality rate of more than $90 \%+7$. Early diagnosis, in conjunction with the introduction of antibiotics to treat the infection, dramatically reduced mortality rates to approximately 15\%; however, significant morbidity remains ${ }^{4 \cdot 7}$. Although LS is a rare disorder, as this paper will highlight, there have been a number of reports indicating it is on the rise. Critical care nurses need to be aware of this syndrome and the association between oropharyngeal infection, neck pain and lung lesions as patients with this syndrome may require critical care treatment. LS must be considered in healthy young adults with a sore throat, neck pain, fevers, rigours and pulmonary symptoms. It requires prompt recognition and effective management to prevent further morbidity or death 3.8 .9 .

This paper examines the extant literature on LS, and provides an overview of the aetiology, pathophysiology, bacteriology, diagnosis and management aspects of this condition. A case study of a young woman who presented to our critical care unit with a severe case of LS is presented to highlight the complex array of issues for critical care staff associated with managing patients with this tare, but certainly not extinct, syndrome.

\section{LITERATURE REVIEW}

Post anginal septicaemia was first described by Courmont \& Cade in 1900 and later described in more detail by Cunningham in $1930^{4}$. However, it was not for several years, through the work of Lemeirre, that the medical profession began to focus attention on this potentially fatal disorder. This was a result of Lemeirre documenting that 18 of the 20 patients who presented to him with post anginal septicaemia subsequently died ${ }^{10}$. In 1936 , he presented these findings to a group of physicians at the Middlesex Hospital Medical. Subsequently, this condition post anginal septicaemia became known as LS+10,11.

LS occurs as a complication of a middle ear infection or pharyngitis ${ }^{10}$. However, a site of dental infection ${ }^{y}$, mastoiditis ${ }^{8}$ and sinusitis ${ }^{12}$ can occasionally be the portal of entry. Moreno, Altozano, Pinalla et al. ' report that the patient typically presents with an extremely sore throat in the initial stage, which is then followed by fever and rigours. These symptoms may also be accompanied by tenderness in the neck with associated swelling.

Sepsis occurs about 3-10 days after the onset of the sore throat ${ }^{13.14}$ and in some cases the pharyngeal symptoms may even resolve prior to sepsis developing '. The infection of the pharyngeal tissue allows the anaerobic organism $F$. necrophorum to drain into the lateral pharyngeal space which contains the internal jugular vein. The anaerobic organisms then cause septic thrombophlebitis of the internal jugular vein ${ }^{4 . x}$.

In 1989 , Sinave, Hardy \& Fardy " undertook a worldwide review of 36 patients diagnosed with LS and found that $97 \%$ of these patients 
developed septic pulmonary emboli. These septic clots may dislodge from the internal jugular vein thrombus, resulting in areas of infected pulmonary infarction ${ }^{10}$. This exhibits as pleuritic chest pain, dyspnoea and occasional haemoptysis ${ }^{12 \cdot 44}$.

The lungs are the most common site of embolic disease as they are considered to be the first pass filter, with chest X-rays (CXRs) showing nodular shadowing and cavitation $49,11 \cdot 12$. According to Lee, Lopez, Genovese \& Loutit ${ }^{10}$, patients may then develop pleural effusion and/or emphysema. Infection may metastasise to other sites including bone, joint and soft tissue, making diagnosis difficult as the syndrome may be confused with right-sided. endocarditis or septic arthritis ${ }^{4,8}$.

In the pre-antibiotic era the syndrome was frequently seen and well recognised. The onset was typical, with clinical presentation of an acute oropharyngeal infection of the tonsils or the peritonsillar tissue and thrombophlebitis of the internal jugular vein, followed by septicaemia and metastatic septic emboli. Within 7.15 days this chain of events usually ended in a fatal outcome. The number of cases reported decreased dramatically in the 1950s, coinciding with the widespread use of penicillin prescribed for the treatment of inflamed painful throats ${ }^{4,7.15}$. However, it is difficult to obtain accurate data on the incidence of LS over the years, as Brazier, Hall, Yuseuf \& Duerden ' postulate that anaerobic bacteriology practised during the 1960s and 1970s were of a poor standard, rendering the recognition of Fusobacterium species in the laboratory problematic.

Lee, Lopez, Genovese \& Loutit ${ }^{10}$ note that since the introduction of antibiotics and the widespread treatment of throat infections, this syndrome is rarely seen today and therefore it is frequently overlooked when it does appear. However, although the syndrome is rare, it is not extinct. In 1998 Reger ${ }^{13}$ alerted us to the finding that since the 1970s there have been 50 reported cases in the western literature of LS. Following this, a retrospective study carried out in England and Wales showed the number of $F$. necrophorum isolates had increased over a period of 10 years, $(1990-2000)$ to a total of $208^{16,17}$. The annual average number of cases reported was 19, but peaked in 1999 at $34^{16,17}$.

The re-emergence of LS may be due to a number of factors, including a reduction in the use of antibiotics for the treatment of sore throats, and/or improvements in microbiology diagnostic techniques within laboratories.

\section{BACTERIOLOGY}

F. necrophorum is a Gram-negative, non-motile anaerobe from the Bacteroidaceae family. It is part of the normal oropharyngeal commensal flora and is also found in the gastro intestinal tract and the female genitourinary tract ${ }^{2,4,4,17}$. Anaerobes are normally seen as the secondary invaders that occur in mixed cultures; however, $F$. necrophorum acts as a primary pachogen in otherwise healthy patients and is usually the only organism isolated from the standard anaerobic blood culture or site of infection ${ }^{4,7,1, i 4}$.

F. necrophorum differs from other anaerobic bacteria, producing a range of toxins similar to non-anaerobic bacteria. These bacteria produce a lipopolysaccharide endotoxin with strong biological activity as well as various exotuxins including leukocidin, haemolycin and hemaglutten. These exotoxins contribute to the platelet aggregation and septic thrombus formation that is related to the pathogenesis of this syndrome ${ }^{2,+, 4, ~}$. Figure I demonstrates the parhogenesis of this syndrome.
F. necrophorum is commonly isolated as the sole pathogen in LS. Other anaerobic organisms less commonly associated with LS include Fusobacterium nucleatum, Fusobacterium gonidiaformans and anaerobic streptococci $i^{\text {8. 10. 19.19. }}$

\section{DIAGNOSIS}

Lemierre himself stated:

The appearance and repetition several days after the onset of a sore throat (and particularly of a tonsillar abscess) of severe pyrexial attacks with an initial rigor, or still more certainly the occurrence of pulmonary infarcts and arthritic manifestations, constitutes a syndrome so characteristic that mistake is almost impossible 4

A diagnosis can be based on clinical findings 14 if thrombophlebitis of the internal jugular vein follows the signs and symptoms of oropharyngeal infection ${ }^{8,10.11}$. The patient will ofren have swelling and tenderness in the upper neck, and may complain of trismus or pain when turning the head away from the side of the thrombus $\$ 10.20$. A CXR showing multiple septic emboli, ultra sound of the neck and computerised tomography (CT) scan of the neck may indicate a thrombosed internal jugular vein confirming the diagnosis of LS. The occurrence of multiple abscesses is also associated with empyema, pleural effusion and pneumothorax 3. ". However, diagnosis can become complicated as the metastatic infection due to bacteraemia can occur also in the brain, meninges, liver, spleen, kidney, muscle, bone, joints, pericardium and endocardium ${ }^{8,1}$. Differential diagnoses for LS includes glandular fever ${ }^{4}$, septic pulmonary emboli' and right sided endocarditis".

The introduction and widespread usage of antibiotics saw a significant decrease in the occurrence of this syndrome ${ }^{4.5,7.11,21}$. As a result, it has been suggested by several authors that physicians may be unfamiliar with detecting the disorder, resulting in delayed or missed diagnosis and/or inappropriate treatment prescribed ${ }^{2,5,7,14}$. Moreover, one study reported that LS is not usually suspected until the pathogen is isolated in blood cultures ${ }^{5}$, which could also be a contributing factor to late or non-detection of the syndrome. However, the growing number of cases of LS sends a warning to physicians managing patients with sore throats to be on the alert for symptoms that may suggest a more serious infection $0.7 \%$.

Figure 1. Pathogenesis of $L S$.

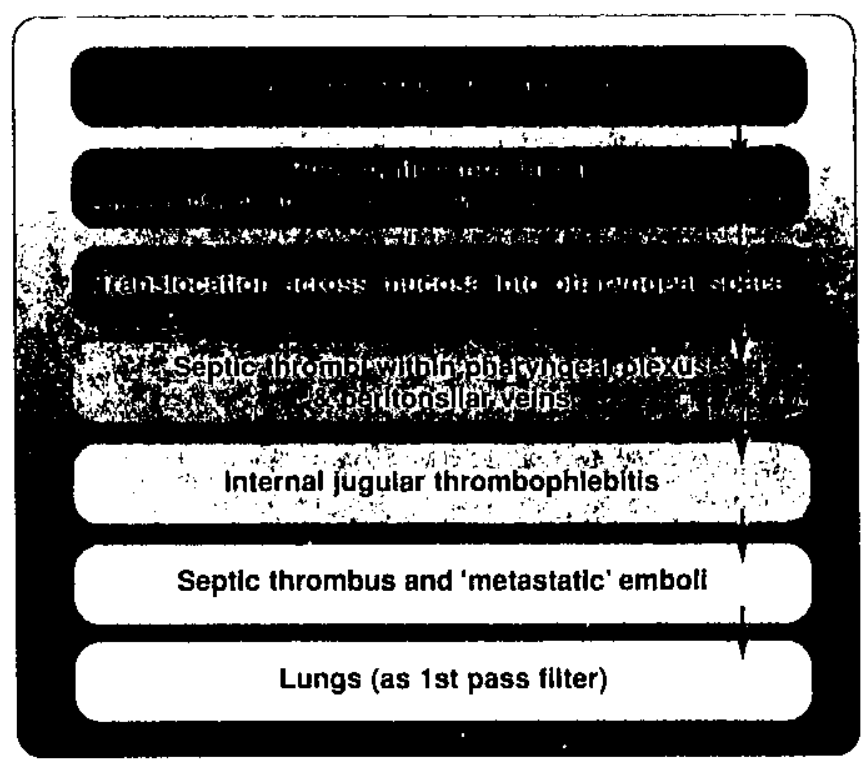




\section{MANAGEMENT}

The recommended treatment of LS includes high-dose intravenous antibiotic therapy from 3-6 weeks to target anaerobic microbes $3.8,15$. Due to the endovascular nature of the infection, prolonged intravenous antibiotic therapy is recommended, as relapses have also been reported with a decrease in the duration of treatment ${ }^{10,11.13}$. Traditionally, $F$. necrophorum has been sensitive to penicillin, clindamycin, metronidiazole and chloramphenicol ${ }^{12.14,20}$. However, Beta (B) lactamase production by some $F$. necrophorum has been reported, thus the use of a $B$ lactamase resistant antibiotic with anaerobic activity such as ticarcillin-clauvulanate is also recommended ${ }^{10,11,13}$.

Moreno et al.'s $1989^{\prime}$ worldwide review of 11 cases of LS reported that all 11 patients received at least 6 weeks of antibiotic therapy and, of these, there were two mortalities. As a result of this finding, Barker, Winer-Muram \& Grey ${ }^{8}$ advocated that antibiotics should not be withheld pending blood culture results as F. necrophorum has a relatively long incubation period of 4 days.

Heparin has also been used as a treatment in LS. Shetty, Begue, Coffman, White-Sims \& Steele ${ }^{3}$ state that the administration of heparin therapy is controversial, a view shared by Barker, WinerMuram \& Grey", who note that although some physicians advocate anticoagulation, there are no control trials available to establish the efficacy of such therapy. Lee, Lopez, Genovese \& Loutit ${ }^{10}$ also agree that the use of anticoagulation in LS syndrome is controversial; nevertheless, they suggest that anticoagulation may expedite improvement and should be considered in the treatment regime when there are no other contraindications. Conversely, the increased risk of haemorrhage and emboli must be weighed against the dissolution of the clot ${ }^{13}$.

Surgical drainage of the abscesses or purulent collections may be necessary in order to alleviate symptoms and obtain specimens for culture $^{3}$. Ligation or excision of the thrombosed jugular vein is not recommended ${ }^{14}$. Ligation was common in the pre-antibiotic period, but generally it is seen as being unnecessary unless septicaemia and septic emboli persist $3,4,9,12,18$.

Although LS is a rarity, its classical presentation is easily recognised. As previously discussed, a delay in the diagnosis and appropriate antimicrobial treatment has resulted in the morbidity and mortality remaining high, at around $15 \%, 8$. Therefore early detection and treatment is essential to reduce adverse effects. The following case study demonstrates the severity of this syndrome, and highlights the importance of early diagnosis and treatment in preventing mortality.

\section{CASE STUDY}

Kristen [this is the patient's real name; she requested we use her correct name and not a pseudonym], a 19 year old female, presented to the emergency department (ED) of a hospital on the Gold Coast, Queensland with a 2 week history of a sore throat and fevers. Prior to this, Kristen had visited her general practitioner every day over the 2 week period, and had been commenced on oral erythromycin as treatment for her symptoms.

On clinical examination in the ED, she was found to have bilateral neck and facial swelling, with a right peritonsillar abscess that was noted to be increasing in size. Kristen was reported to have looked extremely unwell. Her vital signs on admission were: temperature $40.4^{\circ} \mathrm{C}$, pulse tate 96 , respiratory rate 28 , blood pressure $90 / 50 \mathrm{mmHg}, \mathrm{O}_{2}$ saturation on room air $90 \%$. Her chest on auscultation was clear though a portable CXR revealed multiple bilateral opacities. The CXR was shown to the infectious disease consultant who fortunately happened to be in the ED at that time and had seen a case of LS 10 years previously. On reviewing Kristen's history, clinical presentation and CXR, he made the diagnosis of LS.

Following this diagnosis, blood pathology tests were ordered, including blood cultures, full blood count (FBC), erythromycin sedimentation rate (ESR), electrolytes and liver function tests (LFT). Kristen's allergy to penicillin was noted, and she was commenced on intravenous (IV) fluids normal saline, ceftriaxone and metronadiazole. She was also given $1 \mathrm{gm}$ of paracetamol for her high temperature. At this time she was breathing spontaneously via a Hudson mask with $6 \mathrm{~L} \mathrm{O}_{2}$, and her oxygen $\left(\mathrm{O}_{2}\right)$ saturation increased to $98 \%$. Kristen was subsequently transferred later that evening to the Gold Coast Hospital (GCH) intensive care unit (ICU), a large public facility with access to specialist infectious diseases, anaesthetic and intensive care medical and nursing resources.

On arrival at the GCH ICU, an ultrasound scan of Kristen's neck was performed. This showed an internal jugular venous thrombosis extending from the base of the skull $7 \mathrm{~cm}$ in length inferiorly. There was blood flow around the thrombus. This is shown in Figure 2. A CT scan of the head, neck and thorax was ordered for the following morning. Kristen's admitting pathology results were notable for haemoglobin $(\mathrm{Hb}) 87 \mathrm{~g} / \mathrm{L}$, white cell count 10.6 , haematocrit 0.26 , platelets $88 \times 10^{\circ}$, sodium $125 \mathrm{mmol} / \mathrm{L}$ and potassium $2.3 \mathrm{mmol} / \mathrm{L}$. Bilirubin and liver enzymes were mildly elevated. Urinalysis showed $2+$ protein and moderate amounts of occult blood. Following this, clindamycin IV was also prescribed, in addition to the ceftriaxone and metronadiazole already commenced.

The CT scan on day 2 showed a $3 \times 3 \mathrm{~cm}$ peritonsillar collection and a thrombus in the right internal jugular vein, in addition to multiple lung abscesses with some collapse, and bilateral pleural effusion. The results were discussed with the ear, nose and throat (ENT) consultant who scheduled Kristen for the operating theatre (OT) later that evening for drainage of the identified collection and the possibility of a tracheostomy. Her condition was fairly stable prior to OT. Medical staff discussed with her family members the possibility of tracheostomy post surgery and also explained the prognosis of patients with LS.

Figure 2. Day 1: Ultrasound of right internal jugular vein.

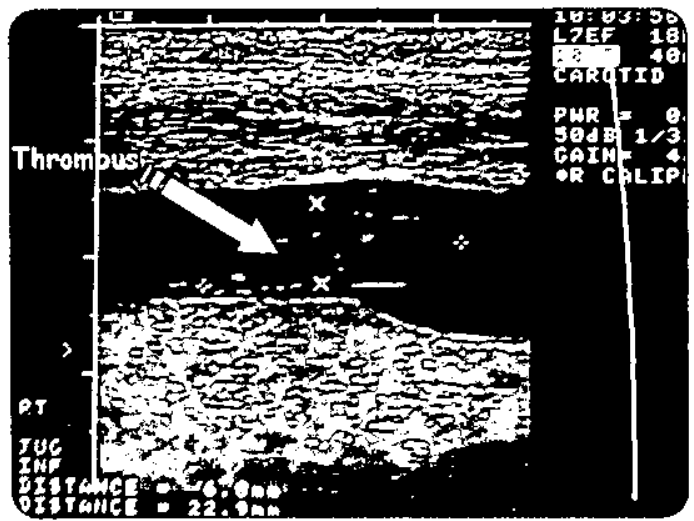


Intra operatively, $50 \mathrm{mls}$ of green offensive pus was drained from the peritonsillar abscess. Kristen returned to ICU following the procedure, and was spontaneously breathing via a Hudson mask at that time. However, her condition deteriorated several hours post operatively. $\mathrm{Her} \mathrm{O}_{2}$ saturation decreased to $88 \%$, she developed a nasal flare and soft stridor, and was noted to have generalised facial oedema and marked trismus (tonic spasms of the muscles of the jaw). She was unable to cough or swallow, and was producing copious amounts of oropharyngeal secretions. Arterial blood gases (ABG) on $15 \mathrm{~L} / \mathrm{min}$ of $\mathrm{O}_{2}$ showed a $\mathrm{pH}$ of $7.23, \mathrm{PaO}_{2} 95 \mathrm{mmHg}$, $\mathrm{PaCO}_{2} 68 \mathrm{mmHg}, \mathrm{HCO}_{3}^{-} 28 \mathrm{mmol} / \mathrm{L}, \mathrm{BE} 0.4$, and $\mathrm{SaO}_{2} 94 \%$. On clinical examination, her epiglottis was oedematous and swollen and there was generalised oedema of the pharynx. Kristen was given nebulised adrenaline prior to being prepared for endotracheal intubation.

During the ensuing days, Kristen's condition worsened. By day 3 , she was peripherally shur down and her oxygen requirements were increasing. $\mathrm{ABG}$ were poor, with a $\mathrm{PaO}_{2}$ of $65 \mathrm{mmH}, \mathrm{PaCO}_{2}$ $38 \mathrm{mmHg}, \mathrm{HCO}_{3}-23 \mathrm{mmol} / \mathrm{L}, \mathrm{BE}-0.7$, on $\mathrm{FiO}_{2}$ of 0.4 . She was also oliguric and hypotensive, with a BP ranging from $89 / 45$. $110 / 60 \mathrm{mmHg}$. Her $\mathrm{Hb}$ was $76 \mathrm{~g} / \mathrm{L}$ and she was transfused 1 unit of packed cells. It was noted that she had pitting oedema around her knees. An IV fluid challenge was prescribed and parenteral therapy was commenced. Kristen's abdomen was distended and intraabdominal pressure recordings were commenced. Readings obtained fluctuated between $16-18 \mathrm{mmHg}$, indicating abnormally high pressures above the norm of $0.5 \mathrm{mmHg}^{21}$. Kristen was found to have extensive abdominal ascites on abdominal ultrasound, which probably accounted for the increased intra-abdominal pressures.

Blood cultures taken on admission were negative at that time. It was later revealed that the blood for culture was only collected in a single aerobic paediatric blood culture bottle at the initial hospital of admission. F. necrophonum would not be expected to grow under such conditions in view of its sensitivity to the presence of oxygen ${ }^{23}$. Brazier, Hall, Yuseuf \& Duerden' report the difficulties in isolating the organism; however, microbiology laboratories with good anaerobic methods are successful in isolating $F$. necrophorum.

The vascular surgeon reviewed Kristen and recommended no surgical interventions, but suggested commencing anticoagulation to prevent extension of the thrombus. In view of the pathogenesis of the disease and risk of haemorrhagic complications, and a review of the literature, the decision was made in consultation with the infectious disease consultant not to anti-coagulate.

Due to the complexity of her illness, the management regimes instigated in the first 8 days of Kristen's clinical path are outlined in Table 1, with the main findings presented.

On day 10 the IV inotropes were ceased as Kristen was maintaining an adequate cardiac output. A surgical tracheostomy was performed on day 11 for the purpose of facilitating long-term mechanical ventilation, and both chest drains were removed. By day 15 Kristen was attempting to communicate with the aid of a spell board (a board with alphabet letters to assist patients who cannot verbally communicate). Kristen was subsequently weaned to pressure support ventilation on day 16 and by day 25 she was breathing spontaneously via a heat moisture exchanger (HME).
Kristen remained febrile throughout this time. Despite appropriate antibiotic coverage, persistent fever is common "s. This is most likely due to the persistent nature of sepsis and bacteraemia ${ }^{12.21}$. On day 29, IV antibiotics were ceased and Kristen was transferred from ICU to the rehabilitation ward where she stayed for only 1 day before being discharged home.

\section{DISCUSSION}

The severity and extent of Kristen's illness episode presented a unique challenge for the ICU staff involved in her management. Caring for a previously healthy young woman with a potentially life-threatening and debilitating illness in an ICU, where the average age of the patients admitted is 54.4 years ${ }^{24}$, was confronting for many. Moreover, as no patients with LS had ever been in the ICU prior to this, the ICU staff, with the exception of the infectious diseases consultanr, were unfamiliar with this syndrome. It was therefore necessary to provide in-service education on LS to enable staff to learn about the syndrome's aetiology, clinical manifestations and management.

Throughout her time in the ICU not only did Kristen require intensive monitoring and physical care, she also needed ongoing emotional support to help her cope with the frustration of impaired communication, being in a foreign environment, and trying to manage the disability associated with the severity of her illness. The nursing staff caring for Kristen remained patient and attentive to her needs, and encouraged her to communicate in non-verbal ways such as writing and using a spell board. Kristen's first attempt at writing was illegible to the nurses; however, she commented at a later date that: "I could read it and was frustrated that no one else could understand $m e "$. As a reminder of the ordeal she endured and survived, Kristen has kept the notes she wrote at that time.

The nursing staff attempted to overcome some of the strangeness of the ICU environment by placing photographs of family members and 'get well' cards where she could easily see them. Llenore \& Ogle ${ }^{25}$ found that critical care nurses' communication with the patient improved when they placed photographs obtained from the patient's family at the bedside. Kristen's parents also brought a personal CD player to allow her to listen to her favourite music, and she commented later that "the music helped everything, and helped pass the time". The nursing staff also organised for Kristen to spend some time outside on the portable mechanical ventilator with her mother, so they could sit together in the sunshine. When judged appropriate, Kristen's mother was encouraged to participate in certain aspects of her care.

The nursing plan therefore reflected and accommodated her psychological needs and encouraged a move towards independence, allowing Kristen to have more control over her environment. This was effective in alleviating some of the frustration for her associated with her illness and dependence on technology. This was an important nursing strategy; Russell ${ }^{20}$ recognised the need for critical care nurses to be aware of patients' rights, and work toward minimising the existing power imbalance in critical care clinical practice.

Initially the tracheostomy tube was a problem for Kristen. According to Chapman ", teenagers are at the stage in life where they are concerned about their appearance. However, Kristen was more concerned with the discomfort of the tube in situ rather than the appearance of the incision site. On discharge from the ICU, 
Day 1 - Admission to GCH ED

$\begin{array}{ll}\text { - Left peritonsillar abscess } & \text { - CXR } \\ \text { - Right tonsillar mass } \uparrow \text { size } & \text { - Blood cultures } \\ \text { - Bilateral neck and tace swelling } & \text { - FBC } \\ \text { - Febrile temp } 40.4^{\circ} \mathrm{C} & \text { - ESR } \\ \text { - BP } 90 / 60 \mathrm{mmHg} & \\ \text { - } \mathrm{O}_{2} \text { sats. on room air } 90 \% & \\ \text { - Respiratory rate } 28 \text { breaths/min }\end{array}$

- $\mathrm{O}_{2}$ therapy $6 \mathrm{~L} / \mathrm{min}$

- IV fluids normal saline

- IV 2gm ceftriaxone

- IV metronidiazole

- 1gm paracetamol

- Transferred GCH ICU

- Respiratory rate 28 breaths/min

Day 1 - GCH ICU

- Admitted ICU

- Reviewed by ENT consultant

- $\mathrm{O}_{2} 6 \mathrm{~L} / \mathrm{min}$

$\begin{array}{ll}\text { - Ultrasound of } & \text { - IJV thrombosis } \\ \text { head and neck } & \text { - Extending from base of skull } 7 \mathrm{~cm} \\ \text { - For CT Scan am } & \text { - Hb } 87 \mathrm{~g} / \mathrm{L} \\ & \text { - Haematocrit } 0.26 \\ \text { - WCC } 10.6 \\ \text { - Plat } 88 \times 109 \\ \text { - Na } 125 \mathrm{mmol} / \mathrm{L} \\ \text { - K } 2.3 \mathrm{mmol} / \mathrm{L}\end{array}$

- KCL $100 \mathrm{mmols}$ added to normal saline/Litre

Day 2

\begin{tabular}{|c|c|c|}
\hline - Severe airway compromise & $\begin{array}{l}\text { - CT scan } \\
\text { - ENT consultation } \\
\text { - Bronchoscopy }\end{array}$ & $\begin{array}{l}\text { - } \mathrm{ABG} \mathrm{pH} 7.23 \\
\text { - } \mathrm{PaO}_{2} 95 \\
\text { - } \mathrm{PaCO}_{2} 68 \\
\text { - } \mathrm{BE} 0.4\end{array}$ \\
\hline - Intubated & - Specimens $\times 2$ & - $\mathrm{HCO}_{3}-28$ \\
\hline $\begin{array}{l}\text { - Ventilated } \\
\text { - Worsening septic picture } \\
\text { - Lines inserted } \\
\text { - Abdomen distended }\end{array}$ & - OT drainage of abscess & - Hb $75 \mathrm{~g} / \mathrm{L}$ \\
\hline
\end{tabular}

- $\mathrm{O}_{2} 15 \mathrm{~L} / \mathrm{min}$

- Nebulised adrenaline commenced prior to intubation

- IV morphine $2.5 \mathrm{mg}$ for pain

- IPPV commenced:

- Fi O2 6 Vt $500 \times 12 \times$ peep $7.5 \mathrm{~cm}$

- IV maintenance fluids

- 2 units packed cells

- Sedated

- Morphine/midazolam

Day 3

- BP 89/45-110/60mHg

- \Urinary output

- Abdominal pressures $\uparrow$

- $\mathrm{FiO}_{2} \uparrow$ Peep $\uparrow$

- Neck and chest mottled

- Peripheries cool

- Pitting oedema to knees

- Femoral CVL inserted

\section{Day 4}

- Remains septic temp 39.1

- Cardiovascular instability

- $\uparrow$ Airway pressures

- $\uparrow \mathrm{O}_{2}$ requirements

- Review by infectious diseases consultant
- IPPV continues

- Inotropes commenced

- Nasogastric feeds commenced @ $20 \mathrm{mls} / \mathrm{hr}$

- $\uparrow$ IV fluids

- $4 \%$ albumex $100 \mathrm{mls} / \mathrm{hr}$

- N/saline $100 \mathrm{mls} / \mathrm{hr}$

- IV antibiotics continue
- Blood cultures negative to date
- IPPV continues

- $\uparrow$ sedation, add propofol

- Commence TPN

- 2 units of packed cells

- Commenced frusemide infusion $5 \mathrm{mg} / \mathrm{hr}$

- Continue Inotropes

- Aim to keep the MAP $>80 \mathrm{mmHg}$

\section{Day 5-6}

- Increasing airway pressures

- Worsening $\mathrm{O}_{2}$ saturation

\section{- CXR}

- Ultrasound neck
- No significant change in size of $\mathrm{Clot}$
- IPPV continues

- N/Saline $\downarrow 50 \mathrm{~m} / / \mathrm{hr}$

- $\uparrow$ PEEP $12.5 \mathrm{~cm}$

- Plan to wean TPN

Day 7-8

- Remains septic

- Abdominal distention persists

- Diarrhoea

- Hepatomegaly \& ascites

- Continue Inotropes

\section{- CXR}

- CT of neck, chest \& abdomen

- Large pleural effusion

- Neck thrombus $46 \mathrm{~mm}$

\footnotetext{
- Hb $111 \mathrm{~g} / \mathrm{L}$

- WCC 12.4

- Plat $155 \times 10^{\circ}$

- INR 1.3

- APTT 32

- Albumin 26

- K 5.1
}

- IPPV continues

- 2 pig tail chest drains inserted under ultrasound

- Drained total 2 litres of straw coloured fluid

- Specimens sent to path 
the tracheostomy scar had faded considerably, much to her disappointment! Kristen joked at the time that she wished that the scar was more prominent as she wanted something to show for her time in $1 \mathrm{CU}$.

Other concerns for Kristen on discharge from ICU related to weight and hair loss. A study by Jones \& Griffith ${ }^{2 *}$ confirms that loss of muscle mass is associated with lengthy stay in ICU and long periods of mechanical ventilation. On going home, Kristen decided to have her hair cut quite short as much of it had fallen out. It took Ktisten at least 6 months to regain the weight she had lost whilst a patient in ICU. Due to the prolonged nature and severity . of her illness, she missed her first semester at university which effectively set her academic progress back one full year. At the present time, Kristen is now in her second year at university, studying creative arts, majoring in contemporary arts and theatre.

\section{CONCLUSION AND IMPLICATIONS FOR PRACTICE}

Although the organism $F$. necrophorum was never isolated on Kristen's blood cultures, the diagnosis and treatment was made on the classical presentation of this syndrome. This case study has been presented to highlight LS, a rare but certainly not extinct disease that continues to be associated with high morbidity and mortality. Although LS is not often seen, patients with sore throats who present to their general practitioner or ED and become more ill following conservative treatment, should be considered for antibiotic therapy and have more extensive laboratory testing performed. Therefore, general practitioners, ED staff and critical care clinicians must be familiar with this potentially lifethreatening condition which necessitates early recognition and prompt initiation of appropriate antibiotic therapy.

Kristen's clinical path was precarious, and we believe that her survival was influenced by early recognition, prompt initiation of treatment and ongoing critical care management. The numerous challenges of Kristen's management were addressed by the ICU nursing and medical staff who collaborated closely with the infectious diseases consultant, microbiologist, radiologist, ENT consultant, vascular surgeons and the allied health tearn. We believe that this was a key factor in ensuring a positive outcome for Kristen, and emphasised the importance of a multi-disciplinary approach to patient care within the ICU.

\section{ACKNOWLEDGEMENT}

The authors would like to thank Dr John Gerrard, consultant infectious disease $\mathrm{GCH}$, for his expert comment on this paper.

\section{REFERENCES}

1. Moreno S, Altuzan JG, Pinalla B, Lopez JC, de Quirus B, Ortega A \& Bouza A. Lemierre's disease: postanginal bacteremin and pulmonary involvement caused by Fusobacterium necropherum. Reviews of Infectious Diseases 1989; 11:319-324.

2. Stahlman GC, DeBuer DK \& Green NE. Fusohacterium osteomyelitis and pyarthrosis: a classic case of Lemierre's syndrome. Journal of Pediatric Orthopaedics 1996; 16(4):529.532.

3. Shetry KA, Begue R, Cofftmin R, White-Sims $S \& \&$ Stecle $R$. Lemierre's syndrome following mastoiditis. Infectious Medicine 1998; 15(5):323-325.

4. Stukroos R, Manni J, de Kruijk J \& Susudijn E. Lemierre's syndrome and acure nustoiditis. Archives of Orolaryngolgy - Head \& Neck Surgery 1999; 125:589-591.
5. Satyanarayana $S$ \& White RL. Fusobacterial infections. The Dalhousie Medical Journal 1999. Available from: URL: http://www.medicine.dal.ca/sdinss/dinj/DMJONLIN/spring99/orig6.htm [10 August 20021.

6. Fiesseler FW. Pharyngitis followed by hypoxia and sepsis: Lemierre's syndrome. The American Journal of Emergency Mecticine 2001; 19(4):320-321.

7. Brazier JS, Hall V, Yuseuf E \& Duerden BI. Fusobacterium necrophorum infections in England and Wales 1990-2000. The Journal of Medical Microbiology 2002; 51(3):269.272.

8. Barker ), Winer-Muram $H \&$ Grey S. Lemierre syndrome. Suuthern Medical Journal 1996; $89(10)$. Available from: URL: http//www.sma.org/smj/96oct $21 \mathrm{htm}$ [10 August 2002].

9. Murray R. Lemierre's syndrome: forgotten, but not gone. 2000. Available from: URL: http://www.usyed.edu.au/ cidm/page/ apro0.hem [10 August 2002].

10. Lee B, Lopez F, Genovese $M$ \& Loutit J. Lemierre's syndrome. Southern Medical Journal 1997; 90(6):640-643.

11. Golpe R, Marin B \& Alonso M. Lemierre syndrome. Postgraduate Medical Journal 1999; 75:141-144.

12. Koay $C B$, Heyworth $T$ \& Burden P. Lemierre syndrome - a forgotten complication of acute tonsillitis. The Journal of Laryngology and Otology 1995; 109:657.661.

13. Lustig LR, Cusick BC, Cheung SW \& Lee KC. Lemierre's syndrome: two cases of post anginal sepsis. Otolaryngology - Head and Neck Surgery 1995; 112:767-772.

14. Sinave $C P$, Hardy GJ \& Fardy PW. The Lemierre syndrome: suppurative thrombophlebitis of the internal jugular vein secondary to oropharyngeal infection. Medicine 1989; 68(2):85.94.

15. Reger $L \&$ Smith $A$. The John Hopkins Microbiology newsletter. The John Hopkins Medical Institutions 1998; 17(44).

16. Jones $J W$, Riordan $T \&$ Morgan MS. Investigation of postanginal sepsis and Lemierre's syndrome in the south-west peninsula. Communicable Disenses \& Public Health 2001; 4:278-282.

17. Feied $\mathrm{C} \&$ Handler J. Thrombophlebitis, septic. Available from: URL: http://www.emedicine.com/emerg/topic581.hem [10 August 2002].

18. Leugers $C M$ \& Clover $R$. Lemierre's syndrome: postanginal sepsis. The Journal of the American Board of Family Practice 1995; $8(5): 384-391$.

19. Gudinchet F, Maeder P, Neveceral P \& Schynder P. Lemierre's syndrome in children: high resolution $C T$ and colour doppler sonography patterns. Chest 1997; 112(1):271.273.

20. Carlson ER Bergamo DF \& Coccin CT. Lemierre's syndrome: two cases of a forgotten disease. Journal of Oral Maxillo Facial Surgery 1994; 5:74-78.

21. Stallworth JR \& Carroll JM. Lemierre's syndrome: new insights to an old disease. Clinical Pediatrics 1997; 36:715-717.

22. Carmel J \& Russo R. Lemierre's syndrome. Chest Supplement, Clinical Case Reports 1997; 112(3):1625.

23. Monaghan W. Lemierre's syndrome: clinical presentation and the role of the microbiology laboratory. Australian Journal of Medical Science $2001 ; 22(2): 72-79$

24. Australasian Outcomes Research Tool for Intensive Care (AORTIC). The National Adult Database for ICU. 1998.

25. Llenore E \& Ogle KR. Nurse-patient communication in the intensive unit: a review of the literature. Australian Critical Care 1999; 12(4):142-145.

26. Russell S. An exploratory study of patients' perceptions, memories and experiences of an intensive care unit. Journal of Advanced Nursing 1999; 29(4):783-791.

27. Chapman $G(E d)$. The Five Love Languages of Teenagers. Sydney: Strand Publishing, 2000.

28. Jones $C \&$ Griffith RD. Ilentifying post intensive care patients who mily need physical rehabilitation. Clinical Intensive Care 2000; $11(1): 35-38$ 